
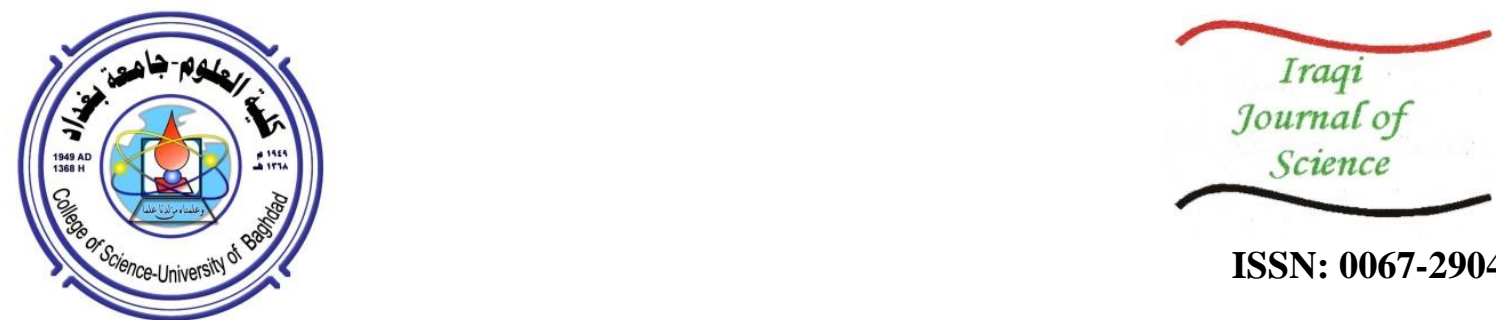

ISSN: 0067-2904

\title{
Capacitance and Resistivity Measurements of Polythiophene /Metallic Nanoparticles-based Humidity Sensors
}

\author{
Estabraq Talib Abdullah, Omar A. Ibrahim \\ Department of Physics, College of Sciences, University of Baghdad, Baghdad, Iraq
}

Received: $1 / 10 / 2020$

Accepted: 16/1/2021

\begin{abstract}
Capacitive-resistive humidity sensors based on polythiophene (P3HT) organic semiconductor as an active material hybrid with three types of metallic nanoparticles (NP) (Ag, $\mathrm{Al}$, and $\mathrm{Cu}$ ) were synthesized by pulsed laser ablation (PLA). The hybrid P3HT/metallic nanoparticles were deposited on indium-tin-oxide (ITO) substrate at room temperature. The surface morphology of theses samples was studied by using field emission scanning electron micrographs (FE-SEM), which indicated the formation of nanoparticles with grain size of about $50 \mathrm{~nm}$. The electrical characteristics of the sensors were examined as a function of the relative humidity levels. The sensors showed an increase in the capacitance with variation in the humidity level. While the resistivity While the resistivity decrease nonlinearity in the variation of humidity level from $10 \%$ to $100 \%$.. The results show that the recovery and response times were higher for the $\mathrm{Al} / \mathrm{P} 3 \mathrm{HT} / \mathrm{Cu} / \mathrm{Al}$ sensor compared with those of the other nanoparticles.
\end{abstract}

Keywords: P3HT, metallic nanoparticles, humidity sensor.

\section{قياسات مقاومة السعوية لأجهزة استثعار الرطوبة من البوليثيوفين / الجسيمات النانوية المعدنية}

\author{
استبرق طالب، عمر عدنان

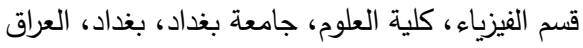

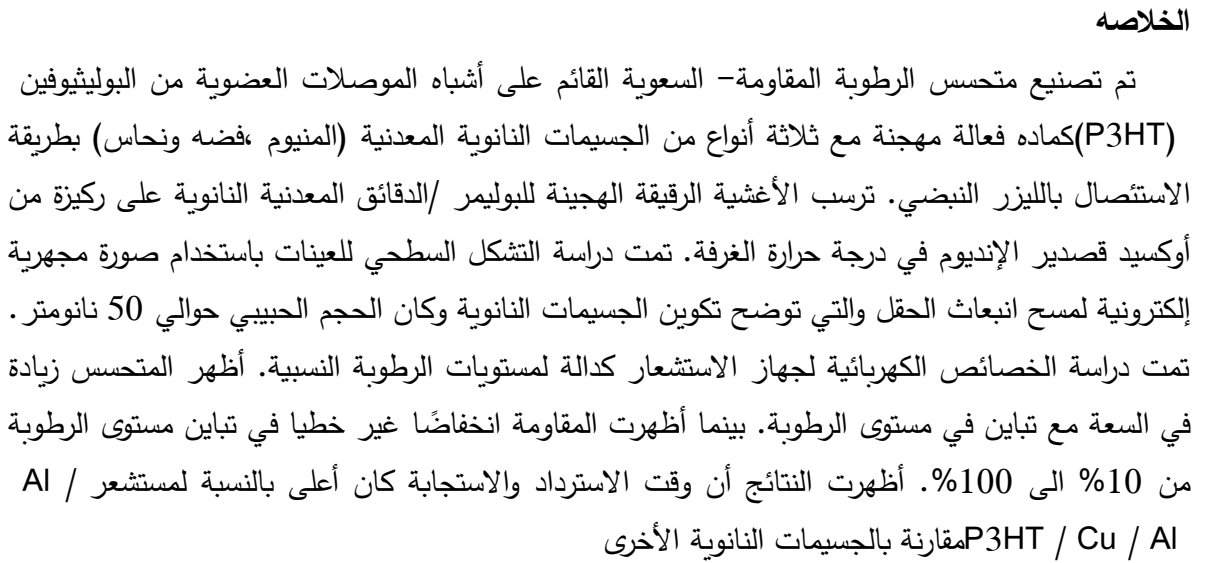

\section{Introduction}

For many years, metal oxide semiconductors were essential to fabricate sensors and used in many applications such as industrial process control, toxic and chemical material detection, and environmental monitoring $[1,2]$. Nowadays, the nanostructure semiconductors are composed in 
sensors to provide better sensitivities for different gases [3, 4]. Their high sensitivities came from their high surface to volume ratio. Despite that, some limitation were recorded, such as high working temperature [5]. In the last decades, the inorganic semiconductors, especially porous silicon have been used extensively for humidity sensing applications,. Nevertheless, the main problems of high cost and high temperature fabrication procedures urged the scientists to search for better materials. Organic semiconductors are a new class of $\pi$-conjugated backbones of macromolecules, where the electrons can move via an $\pi$-electron cloud. They are distinguished by their low cost and easy fabrication. Many mechanisms are involved in controlling the conduction, hopping, tunneling, and mobility gaps of organic semiconductors [6, 7]. Polythiopene (P3HT) was used extensively in transistors, solar cells, and sensors applications due to its elevated hole mobility, good stability, and good electrical conductivity $[8,9]$.

For environmental monitoring and industrial applications, humidity sensors are very important [10]. Humidity sensors can be classified depending on the measuring principle into capacitive, gravimetric, optical, resistive, and integrated types [11]. With a simple comparison between capacitive with resistive humidity sensor, the former show good linearity and greater stability at higher humidity levels. The operation mode of capacitive sensors involves the change in the dielectric constant for the used thin film as humidity sensing occurs with varying humidity levels $[12,13]$. The aim of this work was to increase the sensitivity for humidity by using P3HT with different metallic nanoparticles as an active material in manufacturing $\mathrm{Al} / \mathrm{P} 3 \mathrm{HT} /$ metallic nanoparticles/Al. The effects of varying capacitance and resistance to various humidity levels are tested at different frequencies.

\section{Experimental Part}

Poly(3-hexylthiophene-2, 5-diyl) Regioregular polymer was used as an active material with a molecular weight of $28000 \mathrm{Da}$, supplied by American Dye Source, Inc. Canada. The molecular structure of P3HT is shown in Figure-1. Three metals of high purity were used (Al, $\mathrm{Cu}, \mathrm{Ag}$ ), supplied by the same manufacturer. Chloroform $(\mathrm{CHCl} 3)$ with high purity was used as a solvent. The vacuum chamber was maintained at a pressure of $2 \times 10^{-5}$ mbar throughout the evaporating process. The nanoparticles were synthesized in P3HT solution by laser ablation. Briefly, 0.21g of P3HT was dissolved in $3 \mathrm{ml}$ of chloroform solvent and left overnight to complete the solvation. Metal slices of $1 \mathrm{~cm}^{2}$ dimension were cleaned in ethanol and dried, then placed in $3 \mathrm{ml}$ of P3HT solution and irradiated with a pulsed $(6 \mathrm{~ns}) \mathrm{Nd}: Y A G$ laser at $532 \mathrm{~nm}$, with $10 \mathrm{~Hz}$ repetition rate, 1000Watt, and 20 min irradiation time. To ensure well homogeneous films, spin coating was used with $2500 \mathrm{rpm}$ for 60s. The films were annealed at $60^{\circ} \mathrm{C}$ to remove any remaining solvent. As a substrate, indium-tinoxide (ITO) glass was used in order to manufacture organic semiconductors' surface type capacitor. The ITO substrates with dimensions of $25 \times 25 \mathrm{~mm}$ were washed with acetone and ethanol and ultrasonically placed in deionized water for 20 minutes. After drying, aluminum electrodes with thickness of $150 \mathrm{~nm}$ were thermally deposited on the entire back-side of the substrate, with $50 \pm 5 \mu \mathrm{m}$ gap between them via a shadow mask. The evaporation rate for the deposition was adopted to be 0.3 $\mathrm{nm} / \mathrm{s}$. Then the solution was spin-coated on the ITO substrate with deposited aluminum electrodes with a surface area of $15 \times 5 \mathrm{~mm}^{2}$. After spin-coating, these thin films covered homogeneously the gap between the aluminum electrodes. These samples left to dry for $24 \mathrm{~h}$ at room temperature to form the humidity sensor Al/P3HT/metallic NP/Al.

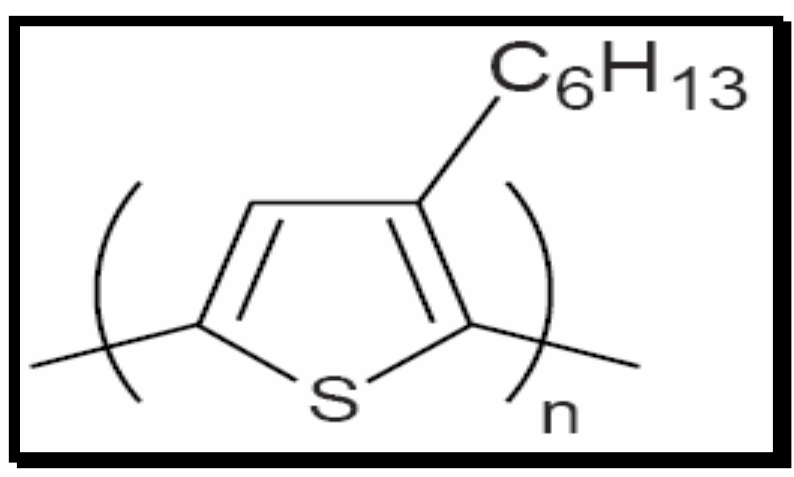

Figure 1-Molecular structure of P3HT [14]. 


\section{Calculation of Humidity Sensor Parameters}

Many parameters affect the capacitance, such as glass substrate area, thickness of the films, and dielectric constant of the materials. Generally, Clausius-Mosotti relation solves the relation between the dielectric constant and polarization $[15,16]$.

$$
\frac{\varepsilon_{d r y}-1}{\varepsilon_{d r y}+1}=\frac{N_{d r y} \alpha_{d r y}}{3 \varepsilon^{\circ}}
$$

where $\varepsilon_{d r y}$ and $\varepsilon^{\circ}$ represents the relative permittivity and the permittivity of free space, respectively, $\mathrm{N}$ is the total number of molecules per unit volume, and $\alpha$ is the molecular polarizability. The relative permittivity can be expressed as:

$$
\varepsilon_{d r y}=\frac{\left(1+2 N_{d r y} \alpha_{d r y}\right)}{\left(1-2 N_{d r y} \alpha_{d r y}\right)}
$$

Also, the same relation can be given for dielectric constant at high humidity

$$
\varepsilon_{H}=\frac{\left(1+2 N_{H} \alpha_{H} / 3 \varepsilon^{\circ}\right)}{\left(1-2 N_{H} \alpha_{H} / 3 \varepsilon^{\circ}\right)}
$$

$\mathrm{N}_{\mathrm{H}}$ and $\alpha_{\mathrm{H}}$ represents the number of molecules per unit volume and the molecular polarizability in humidity, respectively, and can be expressed as

$$
\mathrm{N}_{\mathrm{H}} \alpha_{\mathrm{H}}=\mathrm{N}_{\mathrm{H}} \alpha_{\mathrm{H}}(1+\mathrm{KH})
$$

where $\mathrm{H}$ and $\mathrm{K}$ represent the relative humidity and humidity capacitive factor, respectively. As it is obvious, the value of $\mathrm{N}_{\mathrm{H}} \alpha_{\mathrm{H}}$ depends on the relative humidity level.

The relationship between the dielectric constant and capacitance for humidity is given as:

Now, the humidity sensor can be written as

$$
\frac{C_{H}}{C_{d r y}}=\left(\frac{\varepsilon_{H}}{\varepsilon_{d r y}}\right)^{n}
$$

$$
\frac{C_{H}}{C_{d r y}}=\left(\frac{\left(1+N_{d r y} \alpha_{d r y}(1+K H) / 3 \varepsilon^{\circ}\right)}{\left(1+N_{d r y} \alpha_{d r y}(1+K H) / 3 \varepsilon^{\circ}\right) / \varepsilon_{d r y}}\right)^{n}
$$

\section{Results and Discussion}

Figure-2 shows FE-SEM images of the P3HT/metallic NPs from our previous work [14]. Figure2(a) shows the surface morphology of P3HT NPs which are distributed almost homogeneously on the semiconductor surface. In Figure-2(b, c) shows that the nucleation of the nanoparticles is already formed. During the PLA, the heating process will lead to the release of electrons from the materials in the medium and the formation of more NP atoms. The NPs tend to agglomerate in order to form larger NPs. The NPs surrounded by conduction electrons will lead to a repulsion force that will prevent them from further agglomerating. This result agrees with that of Nayel et al. [11] and Mustafa et al. [14]. The particle size was limited between 20 and $30 \mathrm{~nm}$. This explanation is true for all metallic nanoparticles. For P3HT-Al Np, it was also noted that they are featureless and form strong agglomeration (Figure-2(b). The FESEM images showed clearly that the particles were bound by a surface of amorphous organic residues collected from the polymer decomposition.
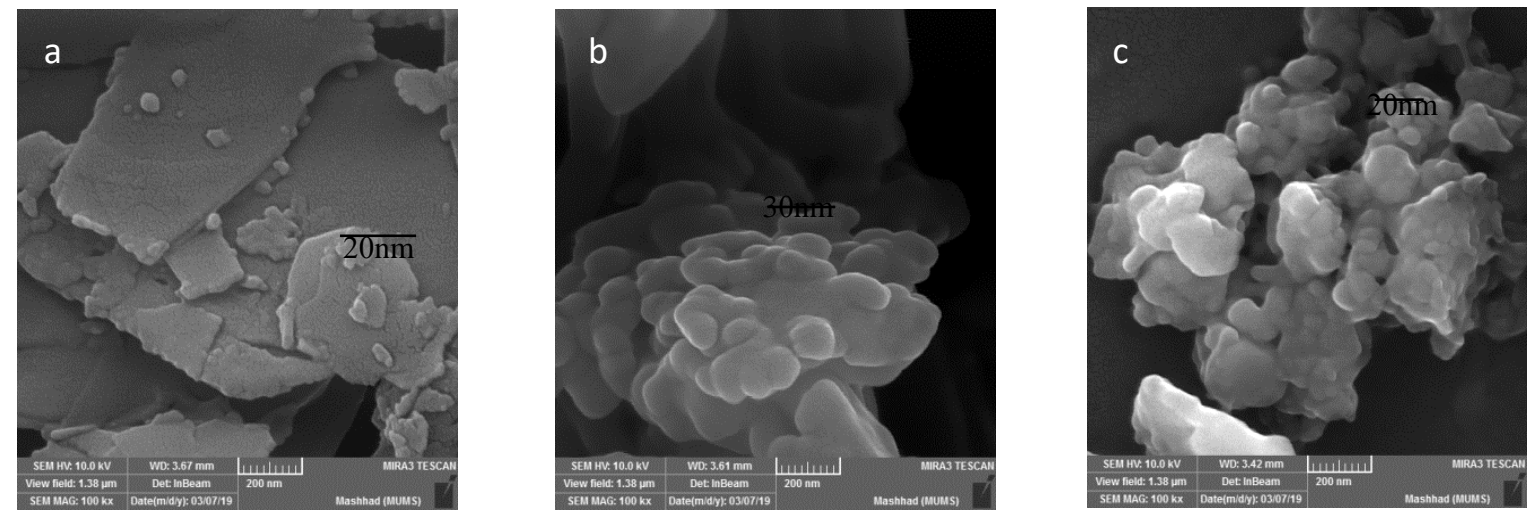

Figure 2-SEM images of P3HT/metallic nanoparticles with size of $200 \mathrm{~nm}$ for (a) Ag, (b) Al, and (c) $\mathrm{Cu}$. 
Figures-3 and 4 illustrate the normalized capacitance $\left(C / C_{0}\right)$ and normalize resistance $\left(R / R_{0}\right)$ values as a function of relative humidity $(\mathrm{RH})$ for frequency $=10 \mathrm{KHz}$ and $\mathrm{T}=16.2^{\circ} \mathrm{C}$. It is clear that the capacitance shows nonlinearity increase in its value for an increase in relative humidity from $10 \%$ to $100 \%$. Such behavior can be attributed to two main factors, which are the absorption of water vapor by the porous medium and the dielectric constant of the organic active material. The absorption is affected by surface roughness and porosity. The rougher and more porous surfaces allow for more absorption sites of water molecules. Increasing humidity level leads to an increase in the absorption of water molecules by the organic active material (P3HT/NP) that finally leads to an increase in capacitance. The dielectric constant of the organic active material is used as a dielectric which adsorbs or desorbs water molecules proportional to the relative humidity, which leads to the increase in the capacitance of the sensor [17]. From Figure-3, the differences in capacitance values for each NP can be observed. This is because of the different dielectric constant values for the different NPs, which are equal to $1.8 \mathrm{eV}$ for $\mathrm{Al}, 6.2 \mathrm{eV}$ for $\mathrm{Cu}$, and $12.2 \mathrm{eV}$ for $\mathrm{Ag}$. This behavior is illustrated by eq.5. Figure-4, which shows the resistivity as a function of relative humidity of the fabricated humidity sensor, where the resistivity is decreased nonlinearly with increasing relative humidity level

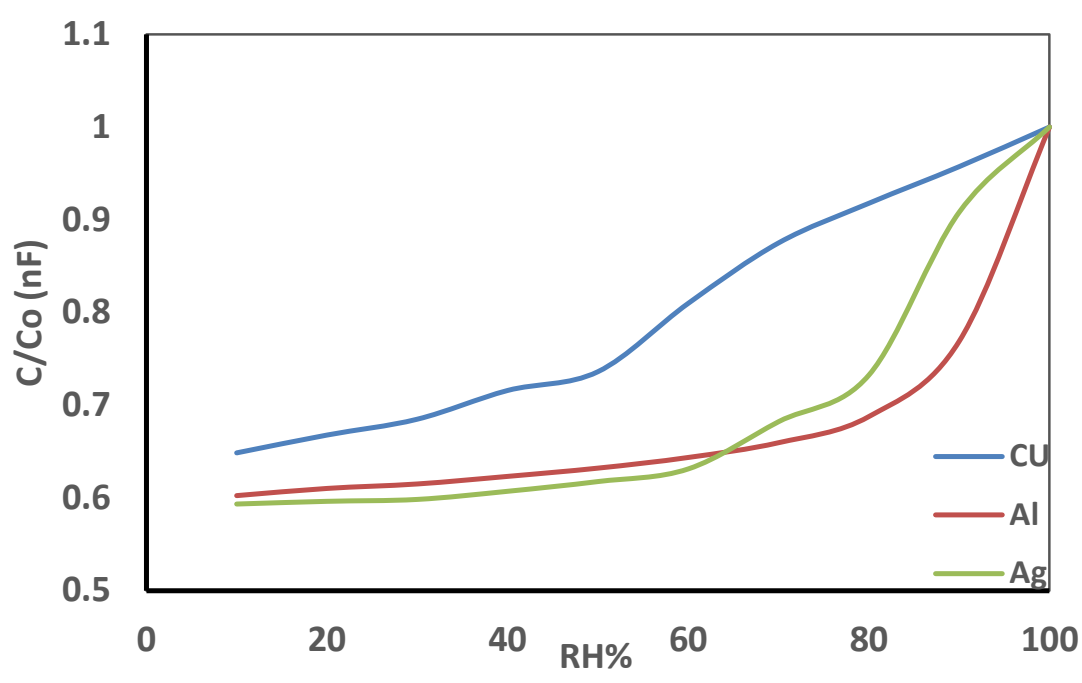

Figure 3-The normalized capacitance $(\mathrm{C} / \mathrm{Co})$ versus relative humidity $(\mathrm{RH} \%)$ for Al/P3HT-metalic NP/Al humidity sensor.

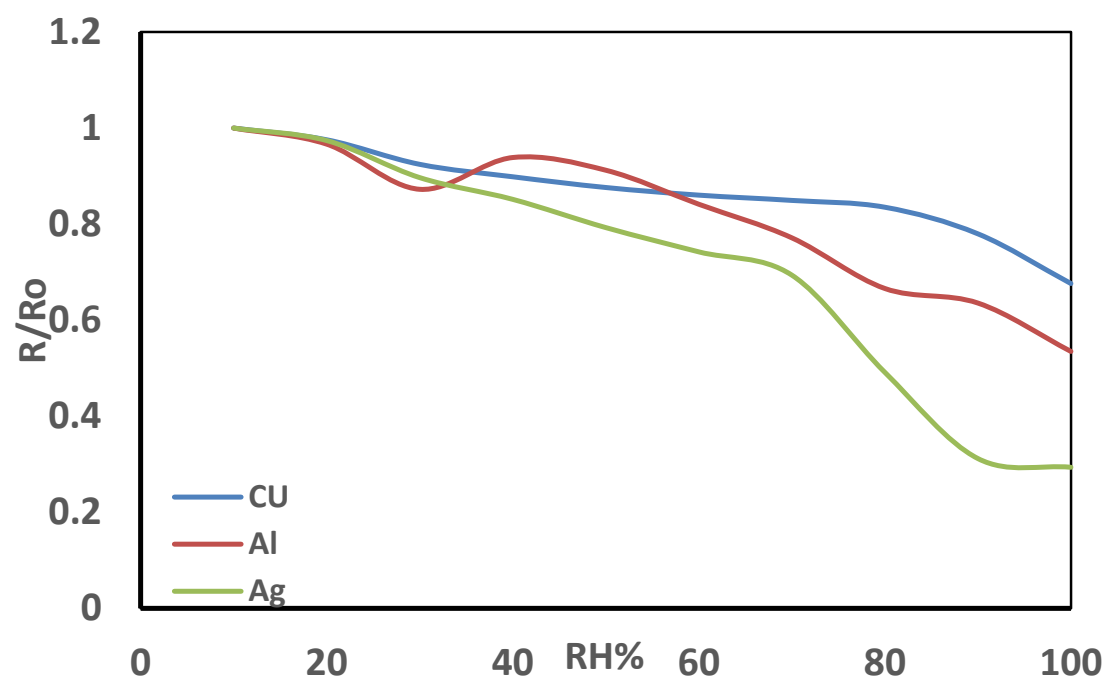

Figure 4-The normalized resistivity (R/Ro) versus relative humidity (RH \%) for Al/P3HT-metalic NP/Al humidity sensor. 
The response and recover time values are essential parameters which determine the use of sensors for different applications. The response time of humidity sensor is the relation between capacitance and time when changing humidity concentration. In general, response time can be determined by measuring the differences in capacitance after realizing the sensor from a maximum to minimum temperature, which is observed by progression in time. For recovery time, it determines when the sensor is suddenly releases from a minimum to maximum temperature.

The response time can be defined as the time required for a sensor output to adjust to $90 \%$ of its final settled value from its previous state. The sensor's recovery time is defined as the time required for the sensor output to decrease to $10 \%$ of the final settled value [18]. The values of response and recovery time for each sensor are illustrated in Table-1. Depending on the experimental results, the Al/ $\mathrm{P} 3 \mathrm{HT} / \mathrm{AlNP} / \mathrm{Al}$ shows a fast response and reasonable recovery time.

Table 1-The response and recovery time values for the used sensors

\begin{tabular}{|l|l|l|}
\hline Sensor & Response Time(s) & Recovery Time(s) \\
\hline $\mathrm{Al} / \mathrm{P} 3 \mathrm{HT} / \mathrm{CuNP} / \mathrm{Al}$ & 37 & 58 \\
\hline $\mathrm{Al} / \mathrm{P} 3 \mathrm{HT} / \mathrm{AgNP} / \mathrm{Al}$ & 28 & 49 \\
\hline $\mathrm{Al} / \mathrm{P} 3 \mathrm{HT} / \mathrm{AlNP} / \mathrm{Al}$ & 26 & 42 \\
\hline
\end{tabular}

\section{Conclusions}

In this work, an organic semiconductor of poly(3-hexylthiophene) (P3HT)- inorganic (three metallic nanoparticles; $\mathrm{Al}, \mathrm{Ag}$, and $\mathrm{Cu}$ ) was produced by pulsed laser ablation (PLA). Drop-casting technique on glass substrates was applied to synthesize the humidity sensor. The performance of this humidity sensor was experimentally studied. The surface morphology shows nanoparticles with a particle size of $50 \mathrm{~nm}$. The capacitance increased while the resistance decreased with increasing humidity level, which is caused by increasing the concentration of water vapor molecules. Al/ P3HT $/ \mathrm{CuNP} / \mathrm{Al}$ sensor shows good stability at higher humidity, compared with resistance humidity sensors. The Al/ P3HT / AlNP / Al shows fast response and reasonable recovery times.

\section{Acknowledgement}

The authors would like to thank Dr. Ameer F. Abdulameer and Dr. Osama N. Najie from the University of Baghdad/ College of Science/Department of Physics for their support during this work.

\section{References}

1. Mohammed,H. R. and Ibrahim, O. A. 2020. Electroluminescence of Light- Emitting Organic Semiconductor/ Europium Oxide Nanoparticle Hybrid Junction. Iraqi Journal of Science, 61(8): 1952-1959. https://doi.org/10.24996/ijs.2020.61.8.12.

2. Najeeb,M. A., Ahmad, Z. and Shakoor, R. A. 2018. "Organic Thin-Film Capacitive and Resistive Humidity Sensors: A Focus Review," Adv. Mater. Interfaces, 1800969: 1-19, 2018, doi: 10.1002/admi.201800969.

3. Ahmad, Z., Zafar, Q., Sulaiman, K., Akram, R. and Karimov, K. 2013. "A Humidity Sensing Organic-Inorganic Composite for Environmental Monitoring”. Sensors, 13(3): 3615-3624. 2013, doi :10.3390/s130303615.

4. Barillaro, G., Lazzerini, G. M. and Strambini, L. M. 2010. "Modeling of porous silicon junction field effect transistor gas sensors: Insight into NO2 interaction," Appl. Phys. Lett., 96(16): 2010, doi: 10.1063/1.3391620.

5. Kruefu,V., Wisitsoraat, A., Tuantranont, A. and Phanichphant, S. 2014. "Gas sensing properties of conducting polymer/Au-loaded $\mathrm{ZnO}$ nanoparticle composite materials at room temperature," Nanoscale Res. Lett., 9(1): 1-12, 2014, doi: 10.1186/1556-276X-9-467.

6. Mohammed, B. H. and Abdullah, E. T. 2020. "Comparison between horizontal and vertical OFETs by using poly (3-Hexylthiophene) (P3HT) as an active semiconductor layer," Iraqi J. Sci., 61(5): 1040-1050, May 2020, doi: 10.24996/ijs.2020.61.5.13.

7. NayelH. H., \& AL-JumailiH. S. 2020. Synthesis and characterization of silver oxide nanoparticles prepared by chemical bath deposition for $\mathrm{NH} 3$ gas sensing applications. Iraqi Journal of Science, 61(4): 772-779. https://doi.org/10.24996/ijs.2020.61.4.9

8. Hamzah,M., Saion, E., Yahya, N., Harun, M. H. Kassim, A. and Mahmud, E. 2007. "Conjugated 
conducting polymers: A brief overview," JASA, 2: 63-68, 2007.

9. Mishra, A. K. 2018. "Conducting Polymers: Concepts and Applications," J. At. Mol. Condens. Nano Phys., 5(2): 159-193, Aug. 2018, doi: 10.26713/jamcnp.v5i2.842.

10. A. F. Abdulameer, M. H. Suhail, O. G. Abdullah, and I. M. Al-Essa, 2017. "Fabrication and characterization of NiPcTs organic semiconductors based surface type capacitive-resistive humidity sensors," J. Mater. Sci. Mater. Electron., 28(18): 13472-13477, 2017, doi: 10. 1007 /s10 854 -017-7186-x.

11. Nayel, H. H. and AL-Jumaili, H. S. 2020. "Synthesis and characterization of silver oxide nanoparticles prepared by chemical bath deposition for NH3 gas sensing applications," Iraqi $J$. Sci., vol. 61(4): 772-779, 2020, doi: 10.24996/ijs.2020.61.4.9.

12. Azmer, M. I., Zafar, Q., Qadir, K. W., Sulaiman, K. Bawazeer T. M. and Alsoufi, M. S. 2018. "Organic humidity sensing film optimization by embedding inorganic nano-anatase $\mathrm{TiO}_{2}$ powder," Applied Physics A, 124(508): 2018, https://doi.org/10.1007/s00339-018-1924-7.

13. Fernandes, L. C., Correia, D. M., Pereira, N., Tubio, C. R. and Lanceros-Méndez, S. 2019. "Highly Sensitive Humidity Sensor Based on Ionic Liquid-Polymer Composites," ACS Appl. Polym. Mater. , 1(10): 2723-2730, 2019, doi: 10.1021/acsapm.9b00675.

14. Ahmed, M. and Abdullah, E. T. 2019. "Synthesis and Characterization of P3HT Metal Nanoparticles Hybrid Junction," Iraqi J. Phys., 17(43): 122-127, 2019, doi: 10.20723/ ijp. 17.43 .122-127.

15. Mitić, V. V., Paunović, V., Lazović, G., Kocić, L. and Vlahović, B. 2018. "Clausius-Mossotti relation fractal modification," Ferroelectrics, 536(1): 60-76, 2018, doi: 10.1080/00150193. 2018. 1528926.

16. Talebian E. and Talebian, M. 2013. "A general review on the derivation of Clausius-Mossotti relation," Optik (Stuttg)., 124(16): 2324-2326, 2013, doi: 10.1016/j.ijleo.2012.06.090.

17. Ahmad, Z., Zafar, Q., Sulaiman, K., Akram, R. and Karimov, K. S. 2013. "A humidity sensing organic-inorganic composite for environmental monitoring," Sensors (Switzerland), 13(3): 36153624, 2013, doi: 10.3390/s130303615.

18. Popov, V. I., Kotin, I. A., Nebogatikova, N. A., Smagulova, S. A. and Antonova, I. V. 2019. "Graphene-PEDOT: PSS humidity sensors for high sensitive, low-cost, highly-reliable, flexible, and printed electronics," Materials (Basel)., 12(21): 1-9, 2019, doi: 10.3390/ma12213477. 